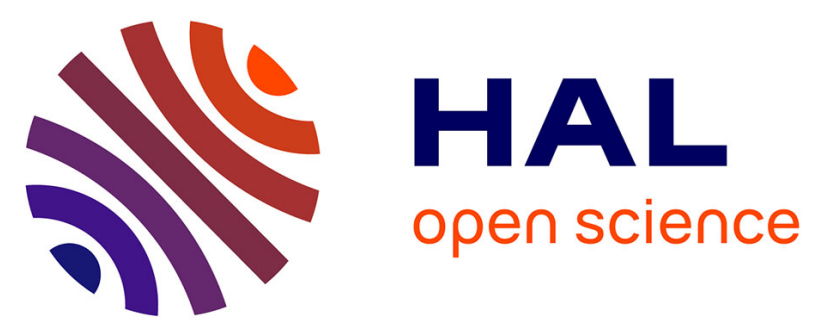

\title{
A new neural network structure dedicated to the control of nonlinear systems
}

Tsurng-Jehng Shen, Felix Mora-Camino, Karim Achaibou

\section{To cite this version:}

Tsurng-Jehng Shen, Felix Mora-Camino, Karim Achaibou. A new neural network structure dedicated to the control of nonlinear systems. MWSCAS 1995, 38th Midwest Symposium on Circuits and Systems, Aug 1995, Rio de Janeiro, Brazil. pp 433-436, 10.1109/MWSCAS.1995.504469 . hal-01022426

\section{HAL Id: hal-01022426 \\ https://hal-enac.archives-ouvertes.fr/hal-01022426}

Submitted on 17 Jul 2014

HAL is a multi-disciplinary open access archive for the deposit and dissemination of scientific research documents, whether they are published or not. The documents may come from teaching and research institutions in France or abroad, or from public or private research centers.
L'archive ouverte pluridisciplinaire HAL, est destinée au dépôt et à la diffusion de documents scientifiques de niveau recherche, publiés ou non, émanant des établissements d'enseignement et de recherche français ou étrangers, des laboratoires publics ou privés. 


\title{
A NEW NEURAL NETWORK STRUCTURE DEDICATED TO THE CONTROL OF NONLINEAR SYSTEMS
}

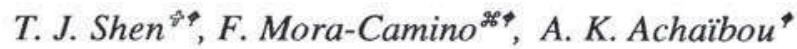 \\ 'L.A.A.S.-C.N.R.S., 7, Av. Colonel Roche, 31077 Toulouse, France \\ ${ }^{2}$ E.N.A.C., 7, Av. Edouard Belin, 31055 Toulouse, France \\ ${ }^{\circledR}$ C.S.I.S.T., Taiwan R.O.C.
}

\begin{abstract}
In this communication is proposed a new neural network structure to design a robust controller for non-exactly known nonlinear systems. Based on input-output data, the neural network structure provides a global affine model of the controlled system which is compatible with well known nonlinear control techniques. An Affine Neural Tracking controller is developed and applied to an inverted pendulum submitted to random perturbations. The performance of the controller and the main advantage of the proposed approach are discussed.
\end{abstract}

\section{INTRODUCTION}

In the last decade nonlinear inverse control theories have been widely developed (input-output linearization). They are known to be especially suited for control tasks such as trajectory tracking and output decoupling. This kind of control approaches is always based on the assumption that precise analytical models of the controlled system are available, and, in addition, an affine model is preferred for the system. But due to practical difficulties of modelisation, the applicability of this class of nonlinear control techniques is often limited.

In this paper, the stabilization problem of an inverse pendulum on a moving car is solved using an inverse control technique combined with a new affine neural network [4]. To apply this combined approach to the considered problem only a priori input-output data relative to the behavior of the pendulum is necessary and no additional formal modelisation is needed.

In the second section of the paper the affine neural network is presented and a procedure to establish an inverse controller applying this affine neural network is shown. In the third section the effectiveness of the proposed approach is displayed by simulation results.

\section{Affine Neural Network AND NeUral Tracking CONTROLLER}

The following approach can be applied to both SISO and MIMO systems, but for the convenience of a simplified presentation, the new neural structure is presented considering SISO discrete-time nonlinear systems.

Let $\Gamma$ be a general input-output model of a nonlinear dynamic system which is supposed to be of the $n^{\text {th }}$ order, square, output feedback controllable and with state observation index $v[2]$, The system output, $y(k+1)$, can be taken as a continuous function of $2(v-1)$ dimensional inputs $y(k), \ldots, y(k-v+1), u(k), \ldots, u(k-v+1)$ :

$$
\begin{array}{r}
y(k+1)=\Gamma[y(k), y(k-1), \ldots, y(k-v+1), \\
u(k), u(k-1), \ldots, u(k-v+1)]
\end{array}
$$

where $u(k)$ is the considered control input for the system.

\section{Affine Neural Approximator}

This neural structure approximates an input-output mapping by the affine form:

$$
\begin{aligned}
\hat{y}(k+1) & =\hat{F}(W, Y(k)) \\
& +\hat{G}(W, Y(k)) \cdot u(k) \quad \forall Y(k) \in U
\end{aligned}
$$

where $\left(\hat{F}(W, Y(k)), \hat{G}(W, Y(k))^{T}\right.$ is the output vector of a neural network while $W$ represents its parameter weight matrices and bias vectors, with $Y(k)=(y(k), \ldots, y(k-v+1), u(k), \ldots, u(k-v+1)) \in \Re^{2(v-1)} \quad$ a n d where $U$ is the operations domain, supposed compact.

DEFINITION. A Multi-layer Neural Network who has $(\hat{F}, \hat{G})$ as output vector and verifies the condition:

$$
\begin{aligned}
& \|\hat{y}(k+1)-y(k+1)\|<\varepsilon \\
& \|\hat{u}(k)-u(k)\|<\varepsilon \quad \forall Y(k) \in U ; \varepsilon \in \Re^{+}
\end{aligned}
$$

is called a Global Affine Neural Approximator of degree $\varepsilon$. If the conditions are verified only on $V \subset U, V \neq U$ then the neural network is called Local Affine Neural Approximator.

Here $\hat{u}(k)=\hat{G}^{-1}(W, Y(k))[y(k+1)-\hat{F}(W, Y(k))]$ where $(u(k), y(k+1)),(\hat{u}(k), \hat{y}(k+1))$ are respectively measured input-output pairs of the system and the approximate values obtained from the neural network defined on $U$.

In [4] a neural network, called $N_{\text {global }}$, of type 
$N_{2(v-1), i_{1}, i_{2}, 2}^{3}$ (Narendra's notation, [2]) with hyperbolic tangent activation functions in the hidden layers and sigmoid functions (with range parameters) in the output layer is shown to be an affine neural approximator of any continuous input-output mapping. The main result which concerns the use of distal teachers, [1], to train a global affine neural approximator is reminded below. Interested readers are referred to [4] for more details.

MAIN ReSUlt: Let $U$ be an admissible domain for a smooth nonlinear function $\Gamma$, let $V_{i}, i=1,2, \ldots, p$ be compact neighborhoods of $p$ input-output pairs defined over $U$ with $V_{1} \cup V_{2} \cup \ldots \cup V_{p}=U$, let $N_{\text {global }}$ be a neural network of type $N_{2(v-1), i, \lambda_{2}, 2}^{3}$ which has $P=\left[Y_{1}, Y_{2}, \ldots, Y_{p}\right]$ as batch training input vectors and $Q=\left[\left(\hat{F}_{1}, \hat{G}_{1}\right)^{T}, \ldots,\left(\hat{F}_{p}, \hat{G}_{p}\right)^{T}\right]$ as batch training target vectors, let $E_{G}=\sum_{i=1}^{p} E_{i}$ be a global error index, then $N_{\text {global }}$ can be trained to be a global affine neural approximator of $\Gamma$, through $p$ parallel distal teachers $N_{D T}^{i}$, $i=1,2, \ldots, p$.

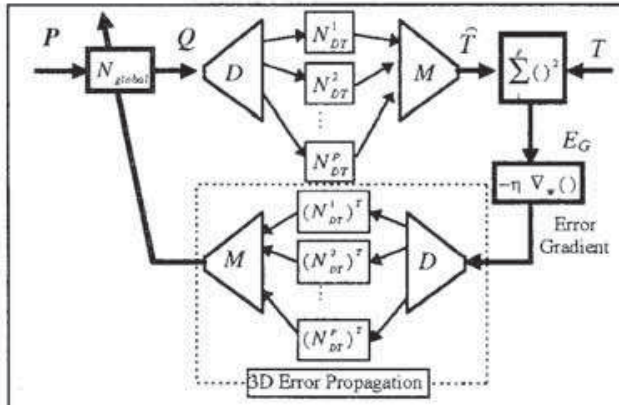

Figure 1. Affine neural network and the learning architecture

\section{3-Dimension Error Backpropagation}

To train $N_{\text {global }}$, a 3-D Error Back-propagation architecture is proposed (Figure 1) where the connections drawn in thick lines represent the classical neural network forward mappings and the error back-propagation paths. $\mathrm{H} \quad$ e $\quad r \quad$ e $\quad T=\left[\begin{array}{cccc}y\left(k_{1}+1\right) & y\left(k_{2}+1\right) & \cdots & y\left(k_{p}+1\right) \\ u\left(k_{1}\right) & u\left(k_{2}\right) & \cdots & u\left(k_{p}\right)\end{array}\right]$, $\widehat{T}=\left[\begin{array}{llll}\hat{t}_{1} & \hat{t}_{2} & \cdots & \hat{t}_{p}\end{array}\right], \hat{t}_{i}=N_{D T}^{i}\left[\begin{array}{l}\hat{F}_{i} \\ \hat{G}_{i}\end{array}\right], i=1,2 \ldots, p$ and $N_{D T}^{i}$ is given by:

$$
N_{D T}^{i}=\left[\begin{array}{cc}
1 & u\left(k_{i}\right) \\
-1 / \hat{G}_{i} & y\left(k_{i}+1\right) / \hat{G}_{i}^{2}
\end{array}\right]
$$

The global error index is defined as $E_{G}=\sum_{i=1}^{p} E_{i}$, where

$$
\begin{aligned}
E_{j}=(1 / 2)\left[\left(y\left(k_{j}+1\right)-\hat{y}\left(k_{j}+1\right)\right)^{2}+\right. \\
\left.\left(u\left(k_{j}\right)-\hat{u}\left(k_{j}\right)\right)^{2}\right]
\end{aligned}
$$

In Figure 1, $D$ represents the decomposition function of a $p$ columns matrix into $p$ separate vectors while $M$ is the inverse function.

\section{Affine Neural Tracking (ANT) Controller}

Once the input-output relation of a system is approximated by an affine form, inverse control techniques (input-output linearization) can be applied.

If the considered system is approximated by the following model :

$$
y(k+1) \cong \hat{F}+\hat{G} u(k) \quad \forall Y(k) \in U
$$

with

$$
\begin{aligned}
\left(\begin{array}{c}
\hat{F} \\
\hat{G}
\end{array}\right) & =N_{\text {global }}(W, y(k), \ldots, y(k-v+1), \\
& u(k), \ldots, u(k-v+1)) \\
& =N_{\text {global }}(W, Y(k))
\end{aligned}
$$

where $N_{\text {global }}: \Re^{2(\mathrm{v}-1)} \rightarrow \Re^{2}$, then the following feedback control law:

$$
u(k)=\widehat{G}^{-1}\left[y_{\text {desired }}(k+1)-\hat{F}\right]
$$

can be used to track the desired trajectory $y_{\text {desired }}(k+1)$.

The corresponding controller is called Affine Neural Tracking (ANT) controller. If $\hat{F}$ and $\hat{G}$ are globally trained over $U$, then the ANT controller is a global nonlinear controller.

It has been shown [4] that either the approximated system is affine or not, the affine neural network can precisely approximate the input-output relation of the system by an affine form for the ANT controller use. Especially, if the controlled system is actually an affine system $(y(k+1)=F(Y)+G(Y) u(k))$, the parameters convergence ( $\hat{F} \rightarrow F ; \hat{G} \rightarrow G$ ) can be easily obtained if the input-output data-base used by training is well designed.

\section{Angle Stability OF INVERTEd Pendulum}

An inverted pendulum mounted on a motor-driven cart is an unstable but controllable system. It can be seen as a model of the attitude control of vertically launched rockets, which, without suitable control action, may fall down any time in any direction.

A simplified two-dimensional inverted pendulum that 
moves only in a vertical (the cart displacement is not of concern) is shown in Figure 2. Here the problem of keeping the pendulum in a vertical position or at a desired inclination angle is treated.

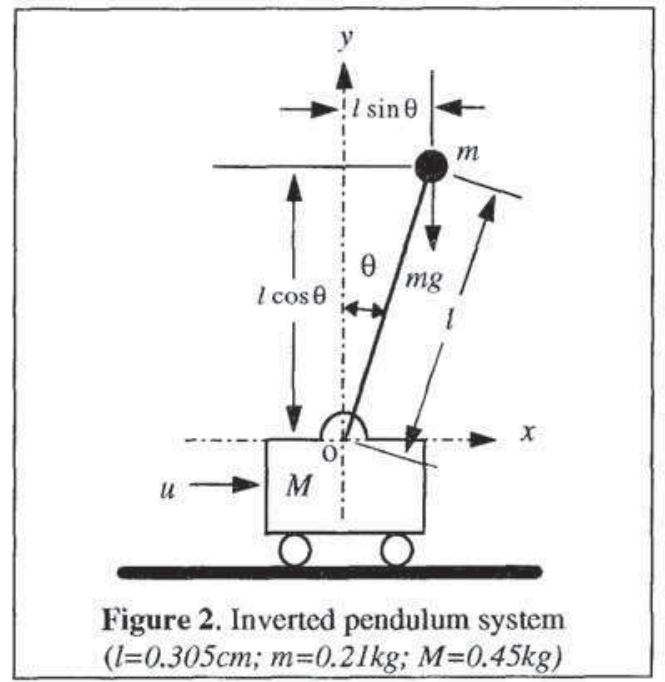

Taking the notations as shown in the above figure and assuming that the pendulum mass is concentrated at the end of a massless rode, the following mathematical model of the system is obtained [3].

$\ddot{\theta}=F(\theta, \dot{\theta})+G(\theta, \dot{\theta}) u$

w i th $F(\theta, \dot{\theta})=\frac{-m l(\cos \theta \sin \theta) \dot{\theta}^{2}+(M+m) g \sin \theta}{l\left(M+m \sin ^{2} \theta\right)}$ a n d

$G(\theta, \dot{\theta})=\frac{-\cos \theta}{l\left(M+m \sin ^{2} \theta\right)}$.

It can be observed that this mathematical model is affine and that if the angular displacement of the pendulum is small, the system can practically be considered as linear:

$$
\ddot{\theta}=\frac{(M+m) g}{M l} \theta+\left(\frac{-1}{M l}\right) u
$$

Since the system model is originally in affine form and $-1 / M l$ is largely different from zero $(-1 / M l \cong 7)$, it is found that the linearized model is precise enough to allow the synthesis of a control law that stabilizes the system. For example, based on (3.2), the linear inverse control law

$$
\begin{aligned}
& u=-M l\left[a\left(\dot{\theta}_{d}-\dot{\theta}\right)+b\left(\theta_{d}-\theta\right)+\right. \\
& \left.c \int_{t_{0}}^{t}\left(\theta_{d}-\theta\right) d t-\frac{(M+m) g}{M l} \theta\right]
\end{aligned}
$$

can easily stabilize the inverted pendulum at any desired angle not greater than $0.8 \mathrm{radian}\left(\left|\theta_{d}\right| \leq 0.8 \mathrm{rad}\right)$. Once the pendulum angle runs out of this range the linearized model becomes too rough to keep the system stable (some other angle regulation examples of the inverted pendulum can be found in [3]).

To show the comparative effectiveness of the proposed approach with respect to linear techniques, the ANT controller will be used to make the system to track various angular positions such that $\left|\theta_{d}\right| \leq 0.8$ radian.

Contrary to the linear approach, in the following development, either the mathematical relations or the physical specifications of the inverted pendulum system are supposed completely unknown. Only some measured input-output data pairs are used to tune an affine neural network, which will provide the necessary affine parameters, $(\widehat{F}, \hat{G})$, to the ANT controller.

To easily get a well excited data base for the training of the affine neural network, the demonstration example of a stabilized inverted pendulum system taken from Matlab/Simulink V.4.0 (a scientific software of MathWorks) is used. This system, being originally stabilized by a classical LQR-controller, was modified to use the above mentioned linear inverse control law. Then with the pendulum angle initialized at $\theta_{0}=-0.5$ radian, the system is made to stabilize at $\theta=0.8$ radian while the system is continuously perturbed by an uniform distributed random force, $-5 \leq u \leq 5.150$ pairs of inputs, $(\theta, \dot{\theta}, u)$, and corresponding output, $\ddot{\theta}$, were successfully recorded during this simulation stage.

An affine neural network of type $N_{2,20,10,2}^{3}$ is then globally trained (off-line) to approximate the pendulum system, while the range parameters in the last layer of the network are chosen such that $-50 \leq \hat{F} \leq 50$ and $-12 \leq \hat{G} \leq-2$.

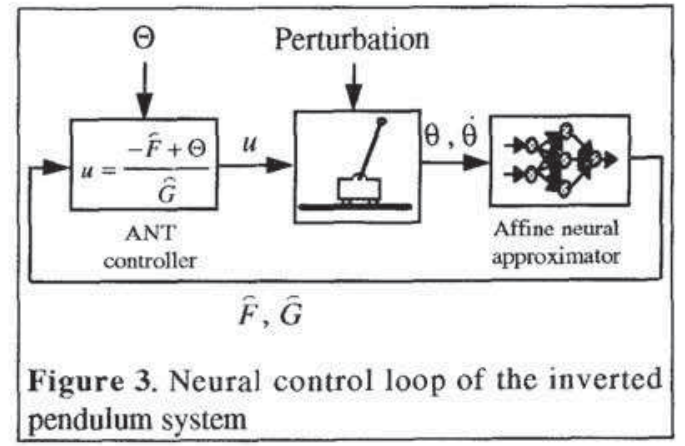

Figure 3 shows the ANT control structure of the inverted pendulum system, where 


$$
\begin{aligned}
\Theta=\ddot{\theta}_{d}+ & 29.28\left(\dot{\theta}_{d}-\dot{\theta}\right)+ \\
& 428.28\left(\theta_{d}-\theta\right)+400 \int_{t_{0}}^{t}\left(\theta_{d}-\theta\right) d t
\end{aligned}
$$

With theses control parameters the error dynamics, $\varepsilon(t)=\theta_{d}(t)-\theta(t)$, of the closed loop system can be expected to be represented by the following linear characteristic equation:

$$
(1+s)\left(s^{2}+2 \rho \omega s+\omega^{2}\right)=0
$$

with $\rho=0.707, \omega=20$ and $s$ is the Laplace variable.

\section{Simulation Results}

In a fifteen-second simulation time, $\theta_{d}$ is successively set to $-0.8,0,0.6,0.8$ and 0 radian, with $\ddot{\theta}_{0}=\dot{\theta}_{0}=\theta_{0}=0$ and $\dot{\theta}_{d}(t)=\ddot{\theta}_{d}(t)=0$. In addition, a random perturbation force uniformly distributed between \pm 5 is applied permanently to the system. Simulation results are shown in Figure 4. It can be seen that the ANT controller make the inverted pendulum to rest at any desired angular position such that $-0.8 \leq \theta_{d} \leq 0.8$.

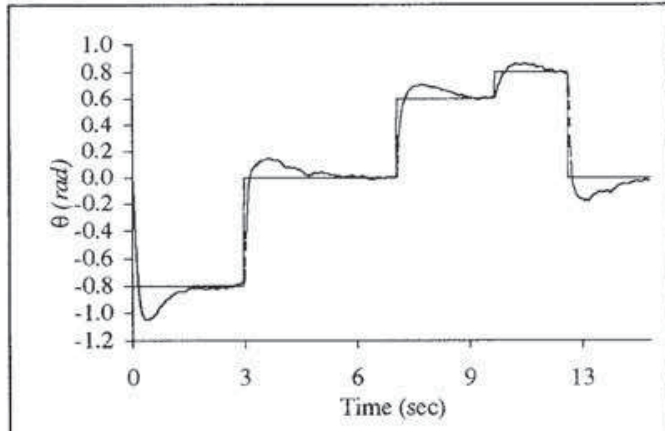

Figure 4. Response of Inverted pendulum system using ANT control, $\left|\theta_{d}\right| \leq 0.8$ radian.

\section{CONCLUSION}

For many simple controllable systems, complicated control techniques are not necessary to get an acceptable control performance, but a suitable model is always needed and, very often, this is the main difficulty or inconvenience to apply well known control techniques.

In this paper has been proposed an Affine Neural Approximator, which can be used to modelize both MIMO and SISO controllable systems in affine form by only referring to input-output measurements of the considered systems. This neural network structure makes feasible the application of inverse control techniques and is specially practical for the control of simple (SISO, affine) systems, for which a neural network can be trained over the whole operation domain with little empirical effort.

An application of this approach - Affine Neural Tracking Controller - has been presented here in the case of the angle-tracking problem of an inverted pendulum (which is SISO and affine). Simulation results show that the ANT controller can easily make the pendulum rest at any angular position over its training domain.

Other much more complex applications have been already considered with success (Aircraft Landing Control, [5]).

In the case where a too large operation domain or a very complex system is considered, on-line training can be user to get a local but adaptive affine neural approximator compatible with the inverse control approach.

\section{REFERENCE}

[1] Jordan M.I., Rumelhart D.E., "Forward Models: Supervised Learning with a Distal Teacher," Cognitive Science, Vol. 16, pp. 307-354, 1992.

[2] Narendra K.S., Mukhopadhyay S., "Adaptive Control of Nonlinear Multivariable Systems Using Neural Networks," Neural Networks, Vol. 7, no. 5, pp. 737-752, 1994.

[3] Ogata K., "Modern Control Engineering", Second Edition, ISBN 0-13-589128-0, Prentice-Hall Inc., 1990.

[4] Shen T.J., Mora-Camino F., Achaïbou A.K., "Output Tracking of Nonlinear Systems Using Affine Neural Controllers" LAAS Report N0. 95033, Toulouse, France, January 1995.

[5] Shen T.J., Mora-Camino F., Achaïbou A.K., "Affine Neural Approximation of Aerodynamic Coefficient Tables, A Control Solution for Aircraft Trajectory Tracking" LAAS Report, Toulouse, France, July 1995. 